\title{
Design and Implementation of a Constant Tension Mid-Upper Arm Circumference (CT-MUAC) device for improved detection of malnutrition in children
}

\author{
Md. Moiful Alam ${ }^{*}$, Rashida Haque, K Siddique-e Rabbani \\ Dept. of Biomedical Physics \& Technology, University of Dhaka, Dhaka-1000, Bangladesh \\ ${ }^{1}$ Presently at Dept. of Electrical and Electronic Engineering, Uttara University, Dhaka-1230, Bangladesh \\ E-mail: moiful03physics@gmail.com, rashida09physics@gmail.com, rabbani@du.ac.bd \\ *Corresponding author
}

\begin{abstract}
Mid-upper arm circumference (MUAC) is an important indicator of the nutritional index, particularly of children in low resource countries of the world. It is a significant anthropometric tool with simple cut-off values which is easy to implement in large scale screening measurements. A conventional MUAC device is essentially an insertion strap and has many merits over other anthropometric measurements techniques, but a lack of reproducibility in measurements is its leading problem since the measurement depends on how strongly a person pulls the strap around an arm of the subject. The aim of the present study is to minimize this reproducibility problem. For this the existing MUAC device has been modified using a short length of an elastic band to provide a constant tension while measuring, making it a Constant Tension MUAC device. Using both the MUAC devices (conventional and CT-MUAC), data were collected from seven subjects by 70 observers (10 observers measuring one subject). Statistical analyses showed that the CT-MUAC device gives a significant improvement with less variability over the conventional device. Thus, the new CTMUAC device has the potential for improved detection of malnutrition throughout the world.
\end{abstract}

Keywords: MUAC, Malnutrition, Reproducibility of MUAC, CT-MUAC.

\section{INTRODUCTION}

Malnutrition of children is a problem in most of the low resource countries, which leads to childhood mortality. Therefore, it is necessary to have simple technologies that help health workers, particularly in rural communities, to identify conditions of malnutrition so that appropriate measures may be taken. A popular and accepted technique is Mid-Upper-Arm Circumference (MUAC), a simple anthropometrical measurement. Human arms basically contain 
muscle mass and subcutaneous fat. Lower levels of muscle mass and subcutaneous fat tend to correspond to a decrease of the mid-upper-arm circumference (MUAC), under the condition of minimized food intake. Thus MUAC measurement can be applied to diagnose malnutrition For which WHO has laid down some threshold values to identify severe and moderate malnutrition for children between 6 and 60 months of age [Myatt et al, 2006, Roberfroid et al, 2013].

Nowadays, mid-upper arm circumference (MUAC) measurement device is nothing but an insertion tape (Figure 1), which is designed to reduce errors of measurement [Zerfas, 1975]. MUAC measurement tape is reasonable in price and comparatively more practical than other soft tissue indices [Visweswara et al., 1970].

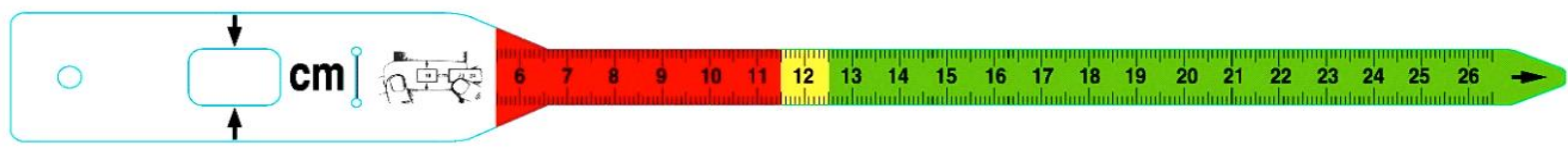

Fig. 1: Conventional strap to measure MUAC

The tape measurement technique was first used in 1958 in community surveys [Jelliffe et al., 1960]. With time the MUAC measurement technique has become more and more dependable index of the nutritional status of children, particularly those aged 'six months to sixty months' [Davis, 1971, Shakir, 1973]. These age groups are 'specific age independent' for mid-upper arm circumference measurement technique [Jelliffe, 1966]. Furthermore, in some cases, comparisons of two indicators (MUAC and weight-for-height) show that they are poorly correlated [Gayle et al., 1988]. MUAC seems to be a better predictor of childhood mortality than height and weight based anthropometric indicators in community-based studies [Vella et al., 1993]. MUAC measurement has commonly been used as a substitute for low weight-for-height (i.e. wasting), which is determined on the basis of a fixed cut-off or threshold value.

In children, a lack of reproducibility in MUAC measurements is the main challenge [Ayele et al., 2012]. One major factor is the tightness of the band when measuring. Since the human body is soft and elastic to some extent, it may be expected that the measured MUAC will depend on the force of pull on the tape by the person making the measurement. Obviously, this factor is expected to 
contribute greatly to a variation of the measurement. In this case, both inter-observer and intraobserver errors in MUAC measurements may occur contributing to both false positive and false negative results when an MUAC threshold value is used to determine whether a child has malnutrition or not. Therefore, a method to reduce such variations in the measurement of MUAC is very important.

The aim of the present work was to explore the effect of a simple but important innovation of our group in order to reduce the variability of MUAC measurement. For this, a simple modification was made to the existing MUAC device to provide a constant tension, for which we have named it a 'Constant Tension MUAC (CT-MUAC)' device. This new device was developed and was used for a comparative evaluation through measurement on several subjects by many observers. It is expected that the new CT-MUAC device may produce more reliable and reproducible results minimizing human errors.

\section{METHODS AND MATERIALS}

\section{Design of the new CT-MUAC device}

The idea for the suggested improvement comes from keeping the tension constant with a simple modification of the existing insertion type strap using a short length of an elastic band. In this design, a short length of an elastic band, as used in dresses, was chosen to provide the constant tension in the existing MUAC device. The MUAC strap was folded at a suitable point and the two ends of the elastic rubber band were glued at a certain distance to the two sides of the fold such that the normal length of the elastic band was less than the corresponding distance on the MUAC strap as shown in Figure 2. Thus when one pulls the two ends of the MUAC strap such that the elastic rubber band elongates to just touch the MUAC strap at all points (Figures 3 and 4), a constant tension will be applied to the MUAC strap since the elastic band extends by a fixed amount. This improvement provided through the elastic band is nothing but an application of Hooke's law, so that a constant tensional force is applied each time for a fixed extension of the elastic band. 


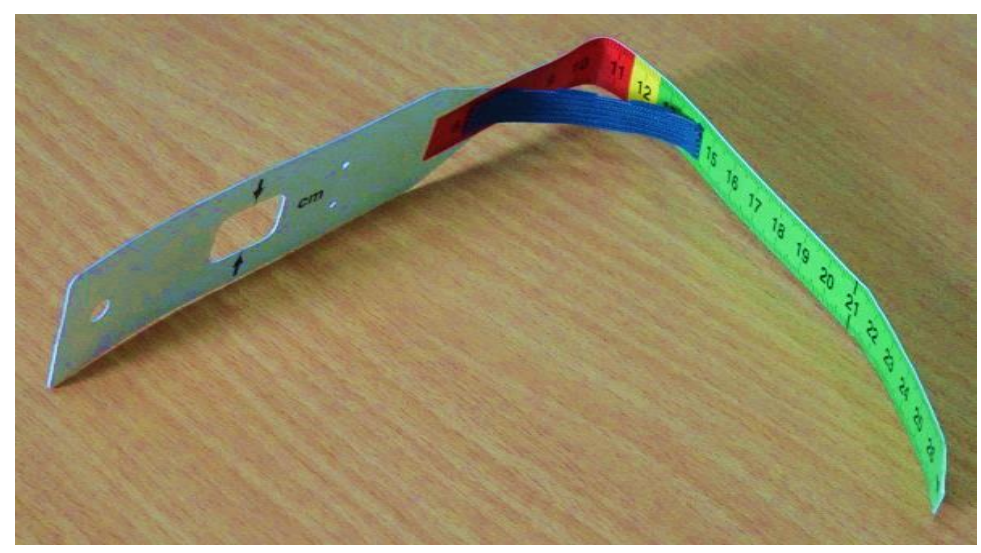

Fig. 2: CT-MUAC strap, modified form of a conventional MUAC strap with an elastic band glued to it at suitable points.

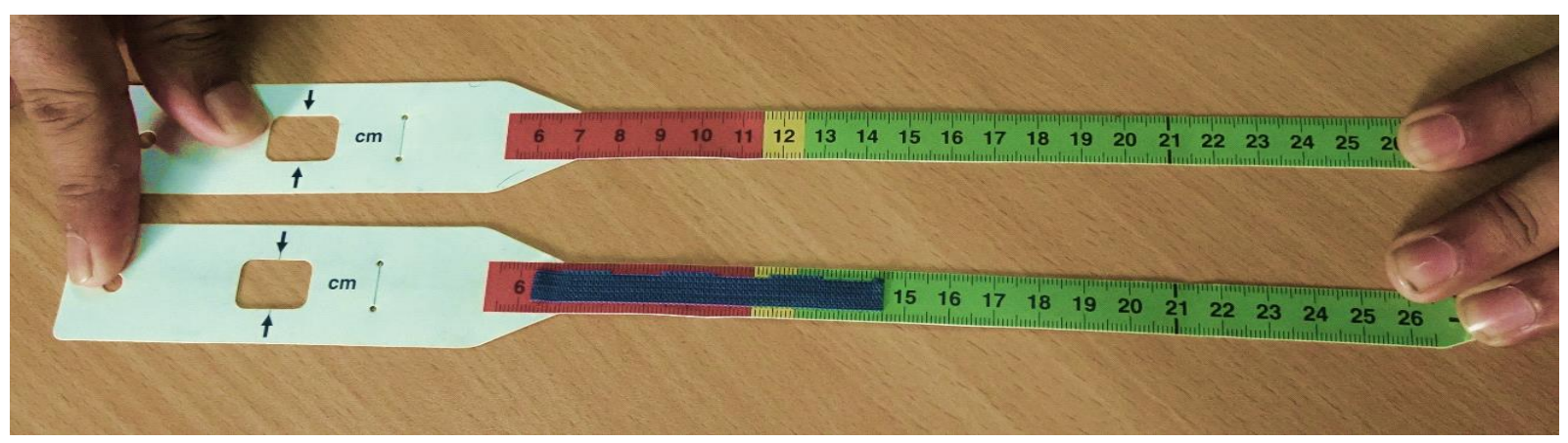

Fig. 3: Elastic band stretched just to touch the MUAC strap in the CT-MUAC device (front). The conventional device is shown at the back

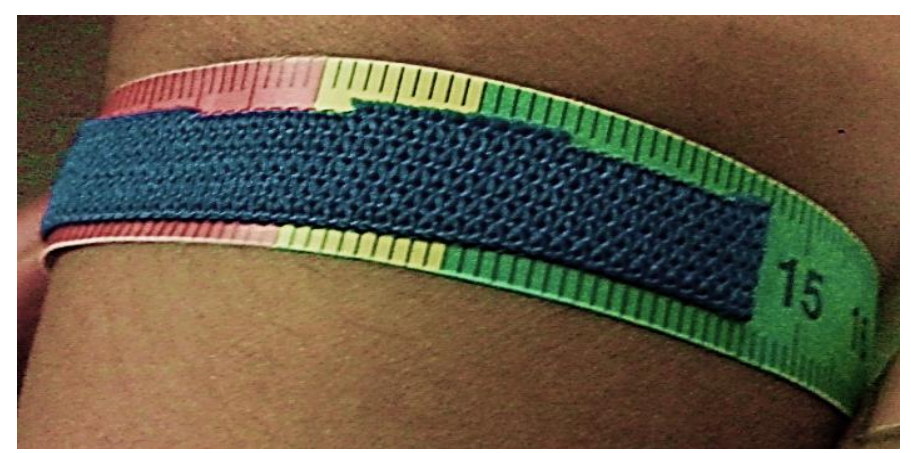

Fig. 4: Elastic band stretched just to touch the MUAC strap in the CT-MUAC device while taking measurements on human subjects 
The measuring process with the new CT-MUAC device is almost the same as the conventional MUAC device except that the observer should make sure that while the elastic band stretches, it just touches the MUAC strap and is not pulled any further (Figure 4).

\section{MUAC Measurement}

MUAC measurements were carried out on only the left upper arm of all subjects. These involved two separate simple measurements, which are: (a) finding the midpoint of the left upper arm from shoulder (lateral acromion) to elbow (distal olecranon), as illustrated in Figures 5-8, and (b) measuring the circumference of the mid-upper arm, as illustrated in Figures 9, 10.

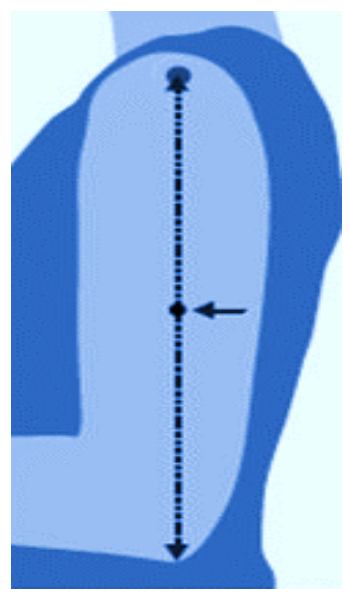

Fig. 5: Length of the left upper arm (geometrical)

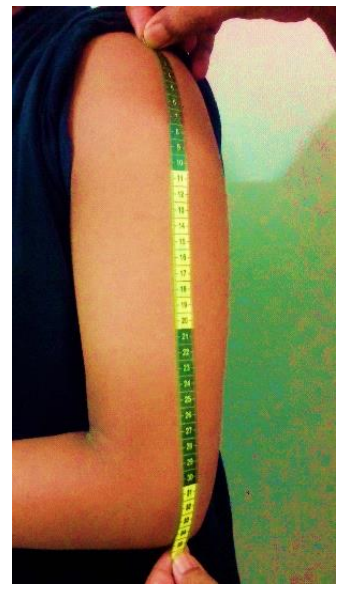

Fig. 6: Length of the left upper arm (practical)

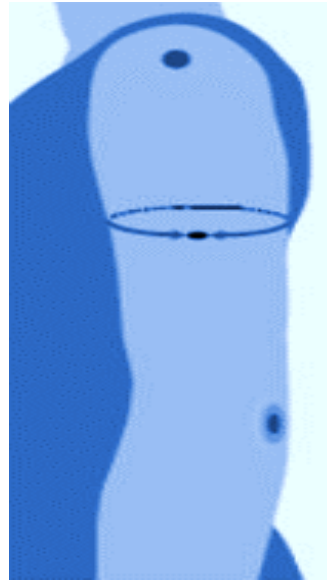

Fig. 7: Midpoint of the left upper arm (geometrical)

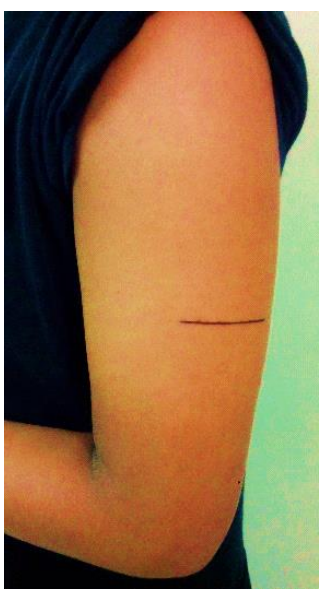

Fig. 8: Midpoint of the left upper arm (practical)

\section{Design of the Reproducibility Study}

The main target of this study is to assess if the new CT-MUAC device gives a better reproducibility. The idea is that for a large number of measurements, dispersion of data would be less for the new device if it gives an improvement. For this purpose, variability of measured values for inter-observer assessments were made using both the conventional MUAC device and the new CT-MUAC device. Inter-observer assessment shows the variability of repeated measurements of the same subject by two or more observers. For the dispersion measurements, the parameters studied are Standard Deviation (SD), Coefficient of Variation (CoV $=\mathrm{SD} / \mathrm{Mean})$, Range (= max value - minimum value) and Percentage Range (\% Range $=$ Range/Mean \%) 
For this study, measurements were carried out on 7 subjects by 70 observers, divided into 7 groups of study. In each group, multiple measurements were taken on a single subject by 10 observers in the particular group using both the MUAC devices (conventional and new). Among the subjects, 5 were adults, 1 was an adolescent and 1 was a child.

\section{Data Collection and Presentation}

For every subject, 10 observers were selected to take the measurements. Each subject and each observer were assigned unique identification numbers. Each observer was briefly trained for a few minutes in the techniques after which he or she was asked to take the measurements. Each observer took 3 to 5 measurements on a single subject using the existing MUAC device and another 3 to 5 measurements using the new CT-MUAC device. This means, 30 to 50 measurements were performed on each subject using each MUAC device by 10 observers. In this way about 400 data points were collected, half of which were taken by conventional MUAC device and another half by the new CT-MUAC device.

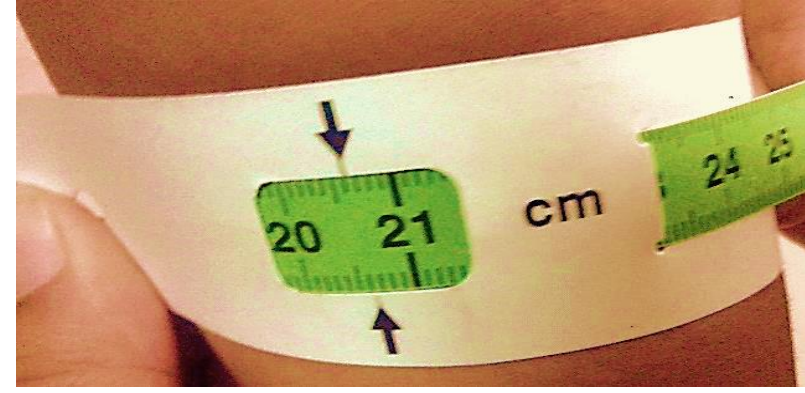

Fig. 9: MUAC measurement using the conventional device (there is no elastic band).

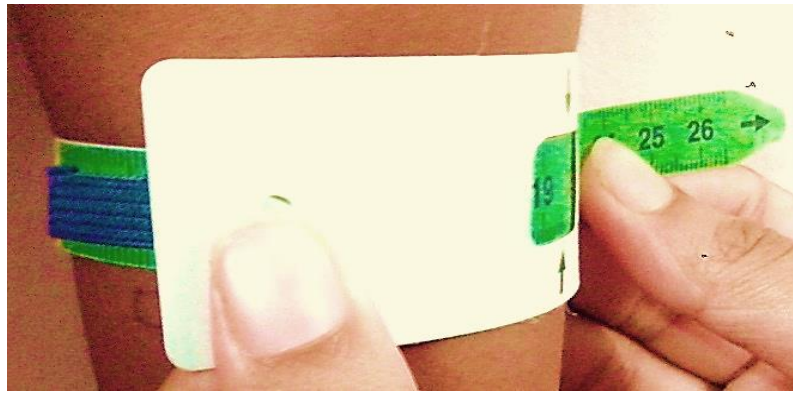

Fig. 10: MUAC measurement using the new CTMUAC device (the elastic band can be seen)

\section{RESULTS AND OBSERVATIONS}

To compare the performances of the two MUAC devices, standard deviation, coefficient of variation (= St Dev/mean), range and percentage range (= range/mean $\%)$ of the values as obtained from the 7 subjects, each tested by 10 separate observers are presented in Table 1. The means of the Coefficient of Variation and the percentage range are also shown for all the data. 
Table 1: Appropriate statistics to compare the measurements using of the two MUAC devices

\begin{tabular}{|c|c|c|c|c|c|}
\cline { 2 - 6 } \multicolumn{1}{c|}{} & \multicolumn{5}{c|}{ Conventional MUAC Device } \\
\hline $\begin{array}{c}\text { Subj- } \\
\text { ect }\end{array}$ & $\begin{array}{c}\text { Mean } \\
\mathrm{mm}\end{array}$ & $\begin{array}{c}\text { St Dev } \\
\mathrm{mm}\end{array}$ & $\begin{array}{c}\text { Coeff of } \\
\text { Variation }\end{array}$ & $\begin{array}{c}\text { Range } \\
\mathrm{mm}\end{array}$ & $\begin{array}{c}\% \\
\text { Range }\end{array}$ \\
\hline 1 & 216.2 & 3.43 & 0.016 & 14 & 6.48 \\
\hline 2 & 229.9 & 3.75 & 0.016 & 12 & 5.22 \\
\hline 3 & 258.9 & 4.84 & 0.019 & 17 & 6.57 \\
\hline 4 & 220.9 & 2.93 & 0.013 & 10 & 4.53 \\
\hline 5 & 224.2 & 3.76 & 0.017 & 15 & 6.69 \\
\hline 6 & 173.5 & 3.40 & 0.020 & 14 & 8.07 \\
\hline 7 & 154.9 & 1.95 & 0.013 & 7 & 4.52 \\
\hline \multicolumn{7}{|c|}{ Mean } & & 0.016 & & 6.010 \\
\hline
\end{tabular}

\begin{tabular}{|c|c|c|c|c|}
\hline \multicolumn{5}{|c|}{ New CT-MUAC device } \\
\hline $\begin{array}{c}\text { Mean, } \\
\text { mm }\end{array}$ & $\begin{array}{c}\text { St Dev, } \\
\text { mm }\end{array}$ & $\begin{array}{l}\text { Coeff of } \\
\text { Variation }\end{array}$ & $\begin{array}{c}\text { Range } \\
\mathrm{mm}\end{array}$ & $\begin{array}{c}\% \\
\text { Range }\end{array}$ \\
\hline 212.0 & 2.26 & 0.011 & 8 & 3.77 \\
\hline 228.2 & 2.80 & 0.012 & 10 & 4.38 \\
\hline 256.6 & 2.39 & 0.009 & 8 & 3.12 \\
\hline 216.8 & 1.75 & 0.008 & 6 & 2.77 \\
\hline 221.4 & 2.12 & 0.010 & 7 & 3.16 \\
\hline 172.3 & 2.04 & 0.012 & 6 & 3.48 \\
\hline 152.4 & 1.63 & 0.011 & 5 & 3.28 \\
\hline \multicolumn{2}{|c|}{ Mean } & 0.010 & & 3.424 \\
\hline
\end{tabular}

The differences of the individual mean values in Table 1, as obtained using the old (conventional) MUAC device and the new CT-MUAC device are shown in Table 2. These are also shown as percentages of the overall mean of all the 14 mean values.

Table 2: Differences of the mean values between measurements taken by the two MUAC devices. The mean of all the 14 mean values obtained using both the devices was used to calculate the percentage difference.

\begin{tabular}{|c|r|c|c|}
\hline Old MUAC & CT-MUAC & & \\
\hline Mean, mm & Mean, mm & Difference, mm & \% difference \\
\hline 216.2 & 212.0 & 4.20 & 2.00 \\
\hline 229.9 & 228.2 & 1.70 & 0.81 \\
\hline 258.9 & 256.6 & 2.30 & 1.10 \\
\hline 220.9 & 216.8 & 4.10 & 1.95 \\
\hline 224.2 & 221.4 & 2.80 & 1.33 \\
\hline 173.5 & 172.3 & 1.20 & 0.57 \\
\hline 154.9 & 152.4 & 2.50 & 1.19 \\
\hline For all 14 data: & & & \\
\hline Mean & 209.87 & & \\
\hline
\end{tabular}


It may be seen that the differences between the mean values obtained using the old and the new MUACs are very small, less than $2 \%$ for all the 7 subjects. This is also apparent from a plot of these mean values in Figure 11.

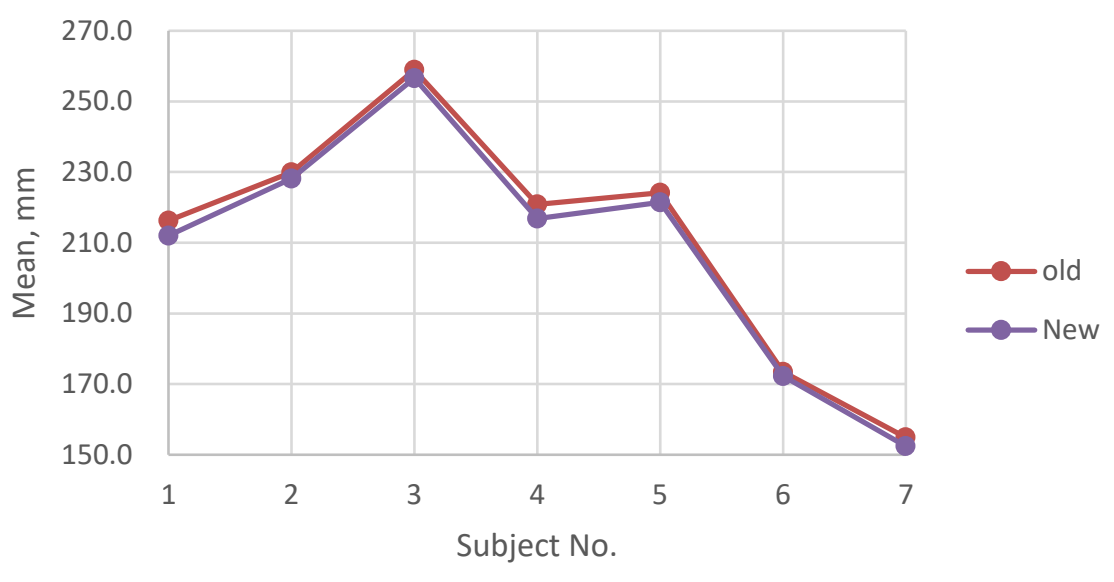

Fig. 11: Plots of the mean values for the 7 subjects obtained using the old (conventional) and the new MUAC devices by 70 observers on 7 subjects (10 observers measuring 1 subject)

However, the dispersion parameters are very different for the two devices, and are plotted in Figures 12-14 using values given in Table 1.

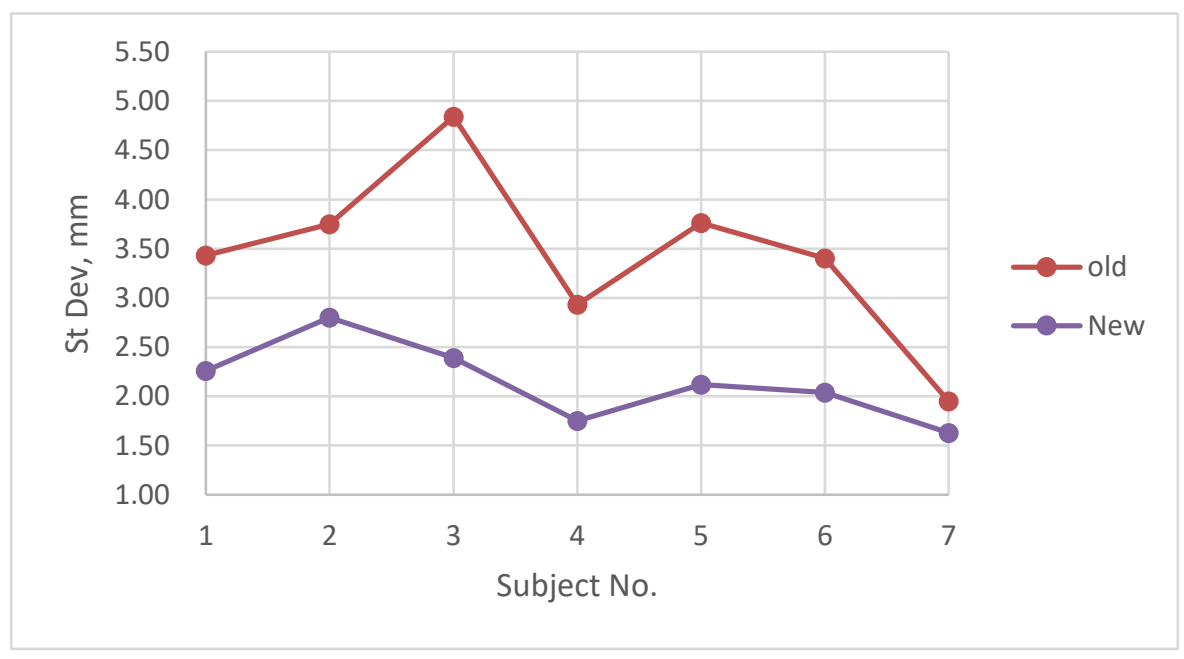

Fig. 12: Standard Deviation (SD) of measurements taken using the two devices. 


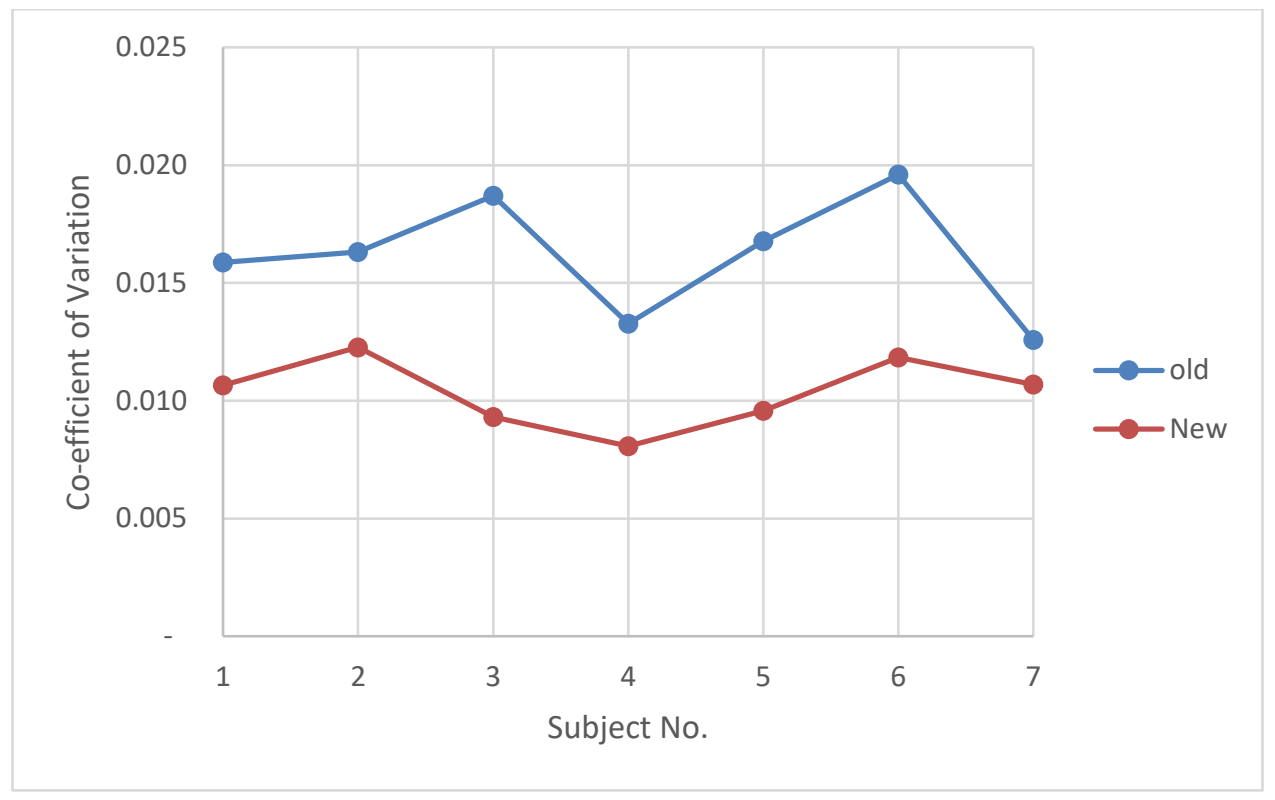

Fig. 13: Coefficient of Variation ( $\mathrm{CoV}$ ) of measurements taken using the two devices

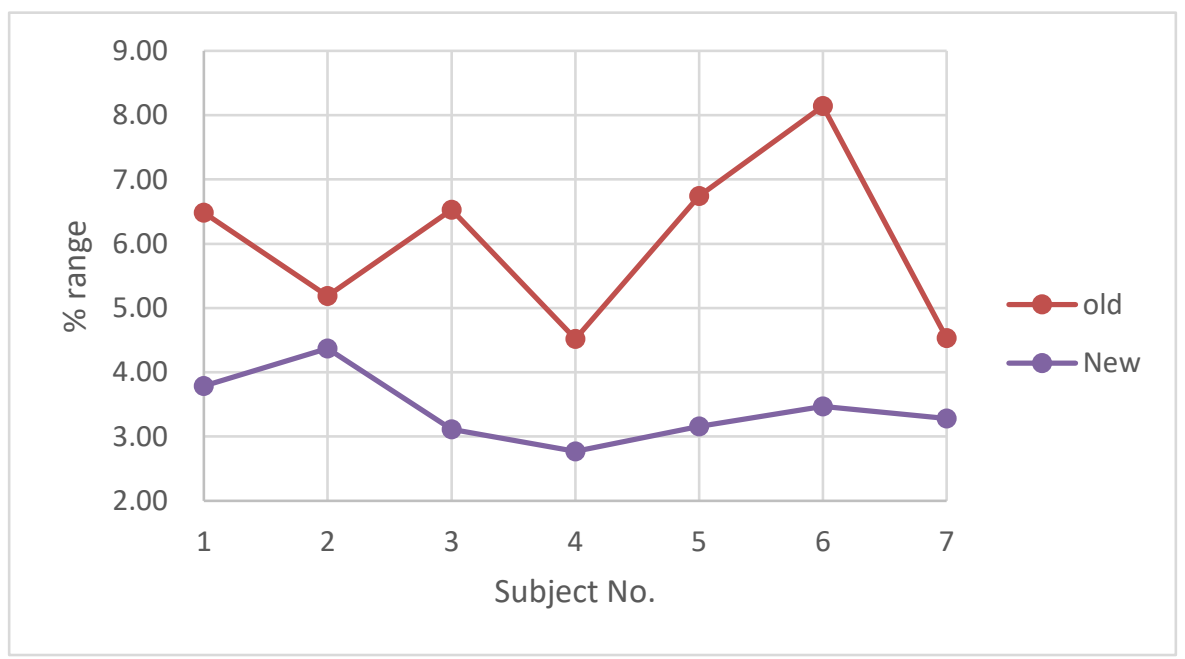

Fig. 14: Percentage of Range of measurements taken using the two devices

The last three figures indicate that all the dispersion parameters are much less in the new CTMUAC device compared to that using the conventional (old) MUAC device. The overall mean values of $\mathrm{CoV}$ and \% Range are reduced to about $62 \%(=0.01 / 0.016 \%)$ and $57 \%(=3.424 / 6.01 \%)$ respectively of those obtained using the conventional MUAC device as deduced from values in Table 1. 


\section{DISCUSSION}

The present study focuses on the reproducibility problem inherent in MUAC measurements, which is widely used for large scale screening for malnutrition in children globally. The problem is more in children since the total circumference is small and a small error in measurement contributes to a greater percentage error compared to that in adults where the circumference is greater. It was identified that the major reproducibility problem may be due to the variation in tension between different measurements, as the arm of a human being is soft and compressible, and the person making the measurement may pull the strap with different strengths at different occasions. Besides, this variability will be more between different observers. This importance of this factor has been proved to a great extent through the measurements reported in this paper. The constant tension has reduced the dispersion parameters considerably.

Therefore, the idea of applying a constant tension is expected to be a marked improvement in the MUAC technique. The proposed CT-MUAC device involves a simple modification of the existing insertion type band using a short length of an elastic band affixed using superglue, and therefore, not a great challenge to adapt worldwide, and it would not increase the cost too much either.

The present comparison study was performed between the existing MUAC device and the new CT-MUAC device on 7 subjects by 70 observers, which is a reasonable size to make the results reliable.

Tables 1 and 2 and Figure 11 shows that the mean values of MUAC obtained using the old and the suggested device was almost identical for all the 7 subjects. As mentioned above, each observer took about 3 to 5 measurements on each subject using each MUAC (old and new) and there were 10 observers making the same measurement on each of these subjects. Therefore, each data point for each of the two graphs in Figure 11 represents the means of 30 to 50 measurements. This apparently suggests that both the devices give the same output and hence there is no point in favour of the new device. However, the improvement becomes clear when one considers the dispersion parameters used in the present study and presented in Table 1 and in Figures 12-14. Each of the dispersion parameters is about 1.5 to 2.5 times less for the new device compared to the old one. This is clearly a significant improvement. Variability in measurements would create many false outcomes when deciding against a fixed threshold in a screening test. Therefore, the less the variability, the better. 
In this device, the only problem of variability would arise if the observer pulls the tape even after the elastic band has reached its maximum length. However, since the observer may easily be trained not to pull any further once the elastic band stretches to its maximum, this variability may be minimized, as is also indicated by the reduced dispersion of the measurements using the new MUAC device. Of course sophisticated feedback techniques may be developed so that further pull is avoided, but that will add much to the complexity, nulling the simplicity of the MUAC technique. Since a person takes extra care in this CT-MUAC naturally, this will act as a natural human feedback to prevent further pull, which has been amply illustrated by the reduced dispersion figures in this work.

Another problem may arise on calibration when making a large number of devices. Besides, an elastic band may lose its elasticity with use and in warm weathers. This aspect needs to be studied in detail before taking up the new CT-MUAC device for mass screening. However, the mean values did not vary much with the two different types of devices as suggested by Figure 11. Therefore, it may be expected that even under such varying situations, the measured values will be useful for screening. The improvement in reproducibility will be there whatever the elasticity may be, since each device will have a constant tension at maximum extension of the elastic band within the timeframe and environment of a set of measurements. Of course the band will need to be replaced if it becomes too loose. This may be done by the health workers themselves given a little training.

These results clearly indicated that the new CT-MUAC device, requiring a very simple and low cost modification to the conventional MUAC device, has reduced variability of measurements considerably. If successfully performed and deployed, the new MUAC device has the potential to improve the detection of malnutrition, particularly of children, throughout the world.

\section{ACKNOWLEDGEMENT}

The authors gratefully acknowledge all of the subjects and observers for their sincere and cordial cooperation during this study. 


\section{REFERENCES}

Myatt, M., Khara, T. and Collins S. (2006). A review of methods to detect cases of severely malnourished children in the community for their admission into community-based therapeutic care programs, $S C N$ Nutrition Policy Paper No. 21, WHO, UNICEF, and SCN Informal Consultation on Community-Based Management of Severe Malnutrition in Children, Edited by Claudine Prudhon et al. Food and Nutrition Bulletin, The United Nations University, 27(3-supplement), pp. S7-S23.

Roberfroid, D., Hammami, N., Lachat, C. et al. (2013). Utilization of mid-upper arm circumference versus weight-for-height in nutritional rehabilitation programmes: a systematic review of evidence. Geneva, Switzerland: World Health Organization (WHO).

Ayele, B., Aemere, A., Gebre, T. et al. (2012). Reliability of Measurements Performed by CommunityDrawn Anthropometrists from Rural Ethiopia, PLoS ONE 7(1): e30345. https://doi.org/10.1371/journal.pone.0030345

Davis, L.E. (1971) Epidemiology of famine in the Nigerian crises: rapid evaluation of malnutrition by height and arm circumference in large populations, The American Journal of Clinical Nutrition, 24: 358.

Gayle, H.D. et al. (1988). Arm circumference versus weight-for-height in nutritional assessment: are the findings comparable, Journal of tropical pediatrics, 34: 213-217.

Jelliffe, D.B. (1966). The assessment of the nutritional status of the community, World Health Organisation, Monograph Ser. no. 53, Geneva, p. 228.

Jelliffe, D.B. and Jelliffe, E.F.P. (1960). Malnutrition in childhood in Haiti, American Journal of Public Health, 50: 1355.

Hooke's Law, Encyclopaedia Britannica. Accessed: 6 November, 2018. https://www.britannica.com/science/Hookes-law.

Shakir, A. (1973). The Quac stick in the assessment of protein-calorie malnutrition in Baghdad, Lancet I, 7 April, pp. 762-763. DOI:10.1016/s0140-6736(73)92142-9

Vella, V. et al. (1993). Anthropometry and childhood mortality in Northwest and Southwest Uganda, American journal of public health, 83: 1616-1618.

Visweswara, R.K., and Singh, D. (1970). An evaluation of the relationships between nutritional status and anthropometric measurements, The American Journal of Clinical Nutrition, $28: 83$.

Zerfas, A.J. (1975). The insertion tape: a new circumference tape for use in nutritional assessment, The American Journal of Clinical Nutrition, 28: 782. 